\title{
I Editorial
}

Im Jahr 2021 ist weiterhin ein Thema zentral: die Sars-Cov-2-Pandemie. Seit Mitte Februar 2020 erfährt die globale Weltgemeinschaft eine Erschütterung, die alle zentralen Themen der Zeit betrifft: Umweltbelastungen und Klimawandel, Veränderungen des natürlichen Habitats und damit zusammenhängende Virusübertragungen von Tier zu Mensch, hohe Arbeitslosigkeit, soziale Ungleichheit sowie Erkrankungen, besonders in Ländern des globalen Südens. Aber auch innerhalb Europas sind Kinder und Jugendliche von einem normalen Schul- und Unterrichtsbetrieb noch weit entfernt, steigen die psychologischen Erkrankungen als Folgewirkungen der Lockdowns und sozialer Isolation. Digitalisierung erfährt einen enormen Vorstoß, allerdings sehr ungleich verteilt zwischen sozialen Gruppen. All diese Themen spiegeln sich auch in unseren bisherigen Veröffentlichungen in Momentum Quarterly wider. Im Jahr 2020 reichten die Themen von plattformbasierter Kurierarbeit über Wohnkosten in Miete oder Eigentum bis hin zu Neokolonialismus in der globalen Ökonomie. Auch Konzepte wie eine Jobgarantie für Langzeitarbeitslose, digitale Identitäten in Zeiten eines Überwachungskapitalismus, die Auswirkungen von Kindergartengebühren auf die Erwerbstätigkeit von Frauen sowie rassismuskritische Überlegungen zur Lehramtsausbildung sind im vergangenen Jahr erschienen.

Insgesamt konnten wir als Herausgeber_innen feststellen, dass zwar vier Frauen in verschiedenen Ausgaben als (Co-)Autorinnen Artikel eingereicht haben, dies aber vermuten lässt, dass die Arbeitsbelastungen im Jahr 2020, Homeschooling und andere Betreuungsaufgaben nicht dazu geführt haben, dass vermehrt Frauen Artikel einreichen. Im Jahr 2019 hatten wir noch einen Frauenanteil von 54 Prozent bei den veröffentlichten Beiträgen, es sind also diesmal deutlich weniger. Wir werden uns weiterhin bemühen, dies zum Positiven zu verändern und besonders jüngere Forscherinnen dazu einladen, bei uns zu veröffentlichen. Stabil blieb hingegen der Anteil an weiblichen Gutachter_innen, er lag 2020 bei 41 Prozent. Die durchschnittliche Zeit von der Einreichung bis zur finalen Entscheidung über die Veröffentlichung eines Beitrags lag 2020 bei rund 100 Tagen. Damit konnten wir die Begutachtungszeiten innerhalb von drei Jahren um über 50 Prozent reduzieren.

Wenn wir nun den Blick nach vorne auf das Jahr 2021 richten, dann eröffnet dieses Editorial den bereits zehnten Jahrgang dieser Zeitschrift. Grund genug, Neues zu wagen. Zum zehnjährigen Jubiläum des Journals werden erstmals zwei Preise vergeben: der Momentum Quarterly Best Paper Award und der Momentum Quarterly Impact Award.

Der Momentum Quarterly Best Paper Award geht an einen besonders herausragenden Beitrag des unmittelbar vorausgehenden Jahrgangs. Der zweistufige Auswahlprozess erfolgt per verdeckter Nominierung durch die Herausgeber_innen und strukturierter Diskussion entlang der vier Qualitätskriterien, an denen sich Momentum Quarterly orientiert: 1. Tragweite der Fragestellung, 2. Theoretischer Beitrag und Kohärenz, 3. Empirische Qualität und innovativer Ansatz, 4. Relevanz für Gesellschaft und Politik.

Der Momentum Quarterly Impact Award richtet sich an jenes Paper, das die größte Wirkung gemessen an Zitierungen entfalten konnte. Als Grundlage dienen alle Zitierungen, die auf Google Scholar mit Stichtag 31.12. des vergangenen Jahres angeführt sind, egal, ob sie sich auf den deutsch- oder den englischsprachigen Titel beziehen. Zitierungen sowohl aus peer-reviewed als auch nicht peer-reviewed Quellen werden summiert, Selbstzitierungen jedoch abgezogen. Die Ergebnisse werden von den Herausgeber_innen mittels Plausibilitätskontrolle überprüft. Bei Gleichstand an Zitierungen entscheidet die Anzahl der Abstract \& Paper-Aufrufe. In Frage kommen alle Arti- 
kel, die vor zehn Jahren in Momentum Quarterly publiziert wurden. Für 2021 sind also alle 2012, im Gründungsjahr der Zeitschrift, publizierten Artikel hinsichtlich ihres Impacts evaluiert worden.

Der diesjährige Momentum Quarterly Best Paper Award geht an die beiden Soziologen Heiner Heiland und Simon Schaupp für ihren hervorragenden Artikel „Digitale Atomisierung oder neue Arbeitskämpfe? Widerständige Solidaritätskulturen in der plattformvermittelten Kurierarbeit“, der in allen vier Kategorien punkten konnte. Angesichts der stetig wachsenden Bedeutung von Online-Plattformen in ganz unterschiedlichen Branchen stellen die Autoren die sehr weitreichende These auf, „dass Digitalisierung nicht allein zur Kontrolle und Atomisierung der Arbeitenden Anwendung findet, sondern von letzteren sowohl zur Schaffung von Solidaritätskulturen als auch zum aktiven Widerstand genutzt wird“ (S. 1). Eingebettet in Literatur zu Kulturen der Solidarität belegen sie diese These auf Basis einer reichhaltigen empirischen Studie (qualitative Interviews, Survey und teilnehmende Beobachtungen), die zeigt, dass trotz Vereinzelung der „Rider“ ausgeprägte Solidarität entsteht, die sich durch gegenseitige Hilfe und herrschaftskritisches Handeln auszeichnet. Dieses kontraintuitive Ergebnis ergänzt akademische Debatten zur wachsenden Kontrolle und Atomisierung von Arbeiter_innen und bietet gesellschaftspolitische Anknüpfungspunkte an Initiativen zur widerständigen Selbstorganisation von Arbeiter_innen in der digitalen Gesellschaft.

Gewinner_innen des ersten Momentum Quarterly Impact Awards sind Karin Fischer und Bernhard Leubolt, deren Beitrag „Auf dem Weg zu mehr Gleichheit? Sozialpolitik in Brasilien und Chile nach dem ,Linksruck“" die meisten Zitierungen aller Beiträge des Gründungsjahrgangs 2012 aufweist. Die Untersuchung zeichnet sich nicht nur durch ihren ganzheitlichen und vergleichenden Ansatz aus, sondern wirkt - im Rückblick aus der Perspektive eines heute beobachtbaren Backlashs in den inzwischen wieder konservativ regierten Ländern - überaus hellsichtig. So werden zwar die gleichheitsfördernden Maßnahmen in beiden Ländern skizziert, gleichzeitig aber die fehlende strukturelle Änderung von Machtverhältnissen (z.B. was Vermögensverteilung und Gewerkschaften betrifft) betont.

Beide Auszeichnungen, der Best Paper und der Impact Award, werden ab sofort jährlich vergeben und im Editorial zur ersten Ausgabe des Folgejahres bekannt gegeben werden. An dieser Stelle möchten wir uns sehr herzlich bei Dennis Tamesberger bedanken, der seit 2015 als Editor von Momentum Quarterly eine führende Rolle eingenommen hat und uns zukünftig mit seiner Expertise als Reviewer unterstützen wird. In diesem Sinne freuen wir uns schon auf spannende Einreichungen, vielleicht auch zum Thema des diesjährigen Momentum-Kongresses: Arbeit.

Leonhard Dobusch, Lukas Lehner, Astrid Mager und Stefanie Wöhl 